\title{
Very-high-energy gamma-ray astronomy
}

\author{
D. Bose ${ }^{1, a}$ and V. R. Chitnis ${ }^{2}$ \\ ${ }^{1}$ Department of Astrophysics and Cosmology, S. N. Bose National Centre for Basic Sciences, Kolkata, India \\ 2 Department of High Energy Physics, Tata Institute of Fundamental Research, Mumbai, India \\ Published online 28 January 2022 \\ (c) The Author(s), under exclusive licence to EDP Sciences, Springer-Verlag GmbH Germany, part of \\ Springer Nature 2022
}

Gamma-rays are the most energetic among all electromagnetic radiation. They account for almost 15 orders of energy in the electromagnetic spectrum, starting from $10^{5} \mathrm{eV}$ and extending beyond $10^{15} \mathrm{eV}$. These energetic photons provide us with a fascinating view of our universe under extreme conditions where the laws of physics are stretched to their limits. Veryhigh-energy (VHE) $\gamma$-rays bring us information from extreme astrophysical objects or phenomena such as neutron stars, black holes, supernova remnants and gamma-ray bursts. The study of VHE $\gamma$-rays is also expected to provide answers to the long-standing puzzle of the origin and acceleration of cosmic rays. In addition, these extremely energetic photons can provide answers about fundamental astrophysical and cosmological aspects such as the extragalactic background light, quantum gravity effect and dark matter. In this special issue, we review the status of ground-based $\gamma$ ray astronomy in two articles. In the first article [1], we discuss the techniques used to detect $\gamma$-rays on the ground, including historical perspective, current status and future prospects. In the second article [2], we present an overview of various astrophysical sources detected by these $\gamma$-ray telescopes to date. We hope that these review articles will be useful for students planning to pursue a career in astrophysics as well as for researchers from various fields.

Gamma-rays are produced by non-thermal processes and follow a power-law spectrum, with flux (number of photons per unit area per unit time) falling steeply with increasing energy. Gamma-ray photons are highly penetrating because of their small wavelengths. However, Earth's atmosphere is opaque to them. At lower energies (a few mega-electron volts to 100 giga-electron volts), $\gamma$-rays can be detected directly using spaceborne detectors. For energies above 100 giga-electron volts, space-based detectors are not adequate for the detection of $\gamma$-rays because of falling flux However, they can be detected using ground-based detectors. A $\gamma$ ray, upon entering the atmosphere, initiates an electromagnetic cascade. The charged particles present in

\footnotetext{
${ }^{\mathrm{a}}$ e-mail: debanjan.bose@bose.res.in (corresponding author)
}

the cascade produce Cherenkov flashes in the atmosphere. As a result, VHE $\gamma$-rays can be detected indirectly by detecting either these Cherenkov flashes or secondary charged particles. Detecting Cherenkov photons or charged particles is not trivial, as one needs to overcome many hurdles. The greatest of these are the backgrounds produced by secondaries initiated by charged cosmic rays in the atmosphere, which are much more numerous than $\gamma$-rays. Cherenkov radiation is emitted in the optical band, and so as in the case of optical telescopes, they can be focused using a mirror and detected by photomultiplier tubes. This method for detecting $\gamma$-rays is called the atmospheric Cherenkov technique. Even though first attempts at detecting VHE $\gamma$-rays were made in the early 1960s, it was the Whipple imaging $\gamma$-ray telescope that established ground-based $\gamma$-ray astronomy on firm footing in the late 1980s. Since then it has been a remarkable journey. The present generation of imaging atmospheric Cherenkov telescopes H.E.S.S. [High Energy Stereoscopic System], MAGIC [Major Atmospheric Gamma Imaging Cherenkov Telescope] and VERITAS [Very Energetic Radiation Imaging Telescope Array System] have taken $\gamma$-ray astronomy to an unprecedented level. The upcoming Cherenkov Telescope Array (CTA) will study the $\gamma$-ray universe with extremely high precision. Even though these telescopes have provided us with abundant information about the high-energy universe, this technique has some inherent limitations. These telescopes can only be operated during clear moonless nights, and they also have smaller fields of view. These limitations can be overcome if, instead of Cherenkov photons, secondary charged particles in the cascade are detected. Several experiments, including HAWC [High Altitude Water Cherenkov] and LHAASO [Large High Altitude Air Shower Observatory], use this technique, known as the extensive air shower technique. These experiments can operate $24 \times 7$ and observe half the sky.

VHE $\gamma$-ray emission has been detected from more than 200 celestial objects to date, and the second article in this issue summarises observations of various classes of these sources comprising both galactic and extra- 
galactic sources. The first source detected at VHE $\gamma$ ray energies is the Crab Nebula, which is a pulsar wind nebula. VHE $\gamma$-ray emission has been detected from various types of galactic objects, such as supernova remnants, pulsar wind nebulae, pulsars and X-ray binaries, along with the important recent discovery of PeVatrons. Amongst extra-galactic sources, VHE $\gamma$-rays have been detected from several active galactic nuclei, gamma-ray bursts and starburst galaxies. Studies of emissions from these objects provide the means to probe extreme environments close to neutron stars and black holes, and to understand characteristics of relativistic jets, winds and explosions. As mentioned earlier, these studies are likely to provide a clue to the origin of cosmic rays as well as inputs towards various fundamental physics aspects. This article gives an overview of the studies carried out so far and also briefly discusses future prospects in the context of CTA and other upcoming projects.

\section{References}

1. D. Bose, V.R. Chitnis, P. Majumdar, B.S. Acharya, Ground-based gamma-ray astronomy: history and development of techniques. Eur. Phys. J. Spec. Top. (2021). https://doi.org/10.1140/epjs/s11734-021-00396-3

2. D. Bose, V.R. Chitnis, P. Majumdar, A. Shukla, Galactic and extragalactic sources of very high energy gamma rays. Eur. Phys. J. Spec. Top. (2022). https://doi.org/ 10.1140/epjs/s11734-022-00434-8 